\title{
Possible effect of SNP TATA-boxes of human erythropoiesis gene promoters on cognitive disorders
}

\author{
Ekaterina Sharypova \\ Molecular Genetics Department \\ Institute of Cytology and Genetics \\ ICG SB RAS \\ Novosibirsk, Russia \\ sharypova@bionet.nsc.ru \\ Mikhail Ponomarenko \\ Systems Biology Department \\ Institute of Cytology and Genetics \\ ICG SB RASNovosibirsk, Russia \\ pon@bionen.nsc.ru
}

\author{
Irina Drachkova \\ Molecular Genetics Department \\ Institute of Cytology and Genetics \\ ICG SB RAS \\ Novosibirsk, Russia \\ drachkova@bionet.nsc.ru
}

\author{
Ludmila Savinkova \\ Molecular Genetics Department \\ Institute of Cytology and Genetics \\ ICG SB RAS \\ Novosibirsk, Russia lksav@bionet.nsc.ru \\ Irina Chadaeva \\ Systems Biology Department \\ Institute of Cytology and Genetics ICG SB \\ RAS \\ Novosibirsk, Russia \\ ichadaeva@bionet.nsc.ru
}

\begin{abstract}
In recent years, there is increasing evidence that various forms of anemia, changes in the quantity and quality of blood cells, may be involved in the pathogenesis of various cognitive disorders accompanying Alzheimer's and Parkinson's diseases, depression of various degrees, etc. In addition to erythroid cells, hemoglobin has been shown to be widely expressed in non-erythroid cells, including neurons of different parts of the brain. We analyzed in silico and in vitro unannotated SNPs in TATA boxes of human genes involved in erythropoiesis. Experimental verification in vitro using the method of electrophoretic mobility shift assay (EMSA) showed the correspondence of prognosis and experimental data. The obtained estimates of the effect of TATA box SNP markers on the formation of TBP/TATA complexes make it possible to consider some SNP markers of erythroid genes as markers of cognitive disorders.
\end{abstract}

Keywords - TATA-binding protein, TATA-box, TBP/TATAaffinnity, erythropoiesis, single nucleotide polymorphism, cognitive disorders

\section{Background}

According to World Health Organization estimates, 7\% of the world population have hereditary aberrations in the synthesis of hemoglobin, meaning that these are the most prevalent monogenic disorders [1]. In recent years, there is increasing evidence that various forms of anemia, changes in the quantity and quality of blood cells, may be involved in the pathogenesis of various cognitive disorders accompanying Alzheimer's and Parkinson's diseases, depression of various degrees, etc. [2]. In addition to erythroid cells, hemoglobin has been shown to be widely expressed in non-erythroid cells, including neurons of the cortex, hippocampus, neurons of the embryos and adult mice brain, and mesencephalic dopaminergic cells of the human, mouse, and rat brain [3]. The morbidity and prevalence of anemia and elevated hemoglobin levels increase with age, which is a heavy burden for society. All this determines the relevance of the problem and the goal of the work, which is to study the effect of SNP TATA boxes of human gene promoters on erythropoiesis and cognitive disorders.

\section{Materials and Methods}

The task was to extract from databases of unannotated single nucleotide polymorphisms (SNP) in the TATA elements of human erythroid genes, to analyze them in silico using the SNP-TATA Z-tester Web service developed at the
ICG SB RAS (http://beehive.bionet.nsc.com/cgibin/mgs/ tatascan_fox/start.pl), the identification of substitutions with potential of functional significance and their experimental verification in vitro using the EMSA method. In vivo, the task is to identify the differential expression of genes involved in erythropoiesis in various parts of the brain of rats domesticated and aggressive towards humans, in order to identify possible candidate genes for cognitive impairment among them.

\section{Results and Discussions}

We analyzed unannotated SNPs in TATA boxes of human globin genes - $H B Z, H B B, H B D, H B G 1$, as well as genes encoding enzymes involved in heme biosynthesis - ALAS1, $C A 1, E P O R, G Y P C$ and others involved in erythropoiesis, a total of 161 SNPs 25 human genes. As a result of the analysis using the SNP-TATA_Z-tester Web service, 45 candidate SNP markers were predicted for 15 of 25 genes that can reliably change the affinity of TBP to the TATA boxes of gene promoters participating in erythropoiesis, and an in silico prognosis of the effect of SNPs on affinity TBP interactions with TATA / TATA-like sequences. Experimental verification in vitro using the method of electrophoretic mobility shift assay (EMSA) showed the correspondence of prognosis and experimental data. As an example, consider the $E P O R$ gene - as you know, the glycoprotein hormone EPO is the main regulator of red blood cell production and exhibits protective properties in cerebral ischemia [4]. To perform the function, it binds to its receptor, EpoR, which dimerizes and activates, which leads to the launch of a cascade of genes responsible for the proliferation, survival and differentiation of erythroid progenitor cells. While EpoR in the blood regulates the differentiation of red blood cells, in the brain it protects several types of neuronal cells from death, including A9 dopaminergic neurons (DA) of the Substantia Nigra (SN) and stores oxygen during hypoxia, which is especially important for neurons with an increased need for energy. In addition to the regulation of erythropoiesis in hematopoietic tissues, where EPOR mutations are the cause of primary hereditary erythrocytosis and are found in $15 \%$ of all cases, erythropoietin is expressed in other tissues, including the nervous system. For functioning, erythropoietin uses a homodimeric receptor (EpoR), which is also widely expressed in the nervous system. The main role of EpoR is to stimulate the proliferation of red blood cell precursor cells for their survival. A 15-fold increase in affinity is caused by the SNP 
of $-27 \mathrm{~T}>\mathrm{A}(\mathrm{rs} 1006576690)$ in the TATA box of the EPOR gene $\left(K_{\mathrm{D}}=170 \pm 30 \mathrm{nM}\right.$ and $11 \pm 3 \mathrm{nM}$.

\begin{tabular}{|c|c|c|c|}
\hline & Gene & \multicolumn{2}{|c|}{ EPOR } \\
\hline \multicolumn{2}{|c|}{$\begin{array}{c}\text { Alleles: } \\
\text { WT or mut }\end{array}$} & WT & $-27 t>A$ \\
\hline \multicolumn{2}{|c|}{$\begin{array}{c}\text { (5'-3'strands), } \\
26 \text { bp }\end{array}$} & ctatttttgt & ctatAttt \\
\hline \multirow{2}{*}{ 苛 } & $-\ln K_{\mathrm{D}}$ & 18.24 & 19.39 \\
\hline & $\delta$ & & +1.15 \\
\hline \multirow{6}{*}{ 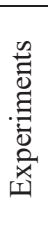 } & $-\ln K_{\mathrm{D}}$ & 15.59 & 18.33 \\
\hline & $\delta$ & & +2.74 \\
\hline & $K_{\mathrm{D}, \mathrm{nM}}$ & $170 \pm 30$ & $11 \pm 3$ \\
\hline & $k_{\mathrm{a}}, \mathrm{M}^{-1} \mathrm{~s}^{-1} * 10^{3}$ & $(2.8 \pm 0,3)$ & $(9.3 \pm 0,7)$ \\
\hline & $k_{\mathrm{d}}, \mathrm{s}^{-1} * 10^{-4}$ & $(4.6 \pm 0.6)$ & $(1.0 \pm 0.3)$ \\
\hline & $\mathrm{t}_{1 / 2}, \min$ & $25 \pm 3$ & $115 \pm 15$ \\
\hline
\end{tabular}

$K_{\mathrm{D}}$ is presented as mean \pm standard deviation; $\delta$ : the difference between the affinity of hTBP for an ODN with and without the SNP in its TATA box expressed as natural $\operatorname{logarithms}, \delta=-\ln \left(K_{\mathrm{D}}, \mathrm{TATA}, \mathrm{Mut}\right)-\left[-\ln \left(K_{\mathrm{D}}, \mathrm{TATA}\right)\right] ; \mathrm{t}_{1 / 2}=$ $\ln 2 / k_{\mathrm{d}}$.

This is accompanied by a 3.3 -fold increase in the rate of formation of complexes $\left(k_{\mathrm{a}}\right)$ and a 4.6-fold increase in their lifetime (or half-life, $\mathrm{t}_{1 / 2}$ ). Several studies have shown that cerebral ischemia leads to increased expression of Epo and EpoR to repair damage. Based on this, it can be assumed that substitutions in the TATA-like sequence of the EPOR gene promoter are $-27 \mathrm{~T}>\mathrm{A},-31 \mathrm{C}>\mathrm{A}$ and $-31 \mathrm{C}>\mathrm{G}$, leading to an increase in the affinity of the TBP/TATA interaction by 15.5 , 8.1 and 2.1 times, which correlates the level of gene expression may indicate a degree of hereditary ischemic brain damage in carriers of minor alleles and may be candidate markers of this disease.

\section{Conclusions}

It is known that hemoglobin genes are expressed in astrocytes of the cortex and hippocampus, in oligodendroglia located in almost all areas of the brain, including the striatum, corpus callosum and medulla oblongata. There are many reports of the association of hemoglobin metabolism disorders with symptoms of mental illness. It is shown that among children with anemia there are significantly more problems with behavior and low intelligence. Many authors believe that cognitive impairment is a causal relationship, and not a consequence of erythropoiesis disorders. Therefore, based on the literature data and the computer-experimental results obtained by us, it can be concluded that the identified candidate SNP markers of erythropoiesis disorders can also be candidate SNP markers of cognitive and mental disorders. Studying the regulatory mechanisms by which detected SNP markers contribute to the development of hemoglobinopathies and associated cognitive impairment will allow doctors not only to take adequate measures to treat hemoglobinopathies in a timely manner, but also to take preventive measures in parallel to prevent or inhibit the development of cognitive and mental disorders. The information received is an additional resource in biomedical research, personalized medicine, diagnostics and drug development.

\section{ACKNOWLEDGMENTS}

The work was supported by the budget project No. 03242019-0042-c-01

\section{REFERENCES}

[1] F.B. Piel, A.P. Patil, R.E. Howes, O.A. Nyangiri, P.W. Gething, M. Dewi, W.H. Temperley, T.N. Williams, D.J. Weatherall, S.L. Hay. "Global epidemiology of sickle haemoglobin in neonates: a contemporary geostatistical model-based map and population estimates". Lancet, vol. 381, pp. 142-151, 2013.

[2] M..A. Altinoz, B. Ince. "Hemoglobins emerging roles in mental disorders. Metabolical, genetical and immunological aspects". J Dev Neurosci, vol. 61, pp. 73-85, 2017.

[3] M. Biagioli, M. Pinto, D. Cesselli, M. Zaninello, D. Lazarevic, P. Roncaglia, R. Simone, S. Vlachouli, C. Plessy, N. Bertin, A. Beltrami, K. Kobayashi, V. Gallo, C. Santoro, I. Ferrer, S. Rivella, C.A. Beltrami, P. Carninci, E. Pavlova, S. Gustincich. "Unexpected expression of alpha- and betta-globin in mesencephalic dopaminergic neurons and glial cells". Proc Natl Acad Sci USA, vol. 106, pp. 15454-15459, 2009. 